\title{
Lattice QCD for Neutrino Programs
}

\section{Michael Wagman}

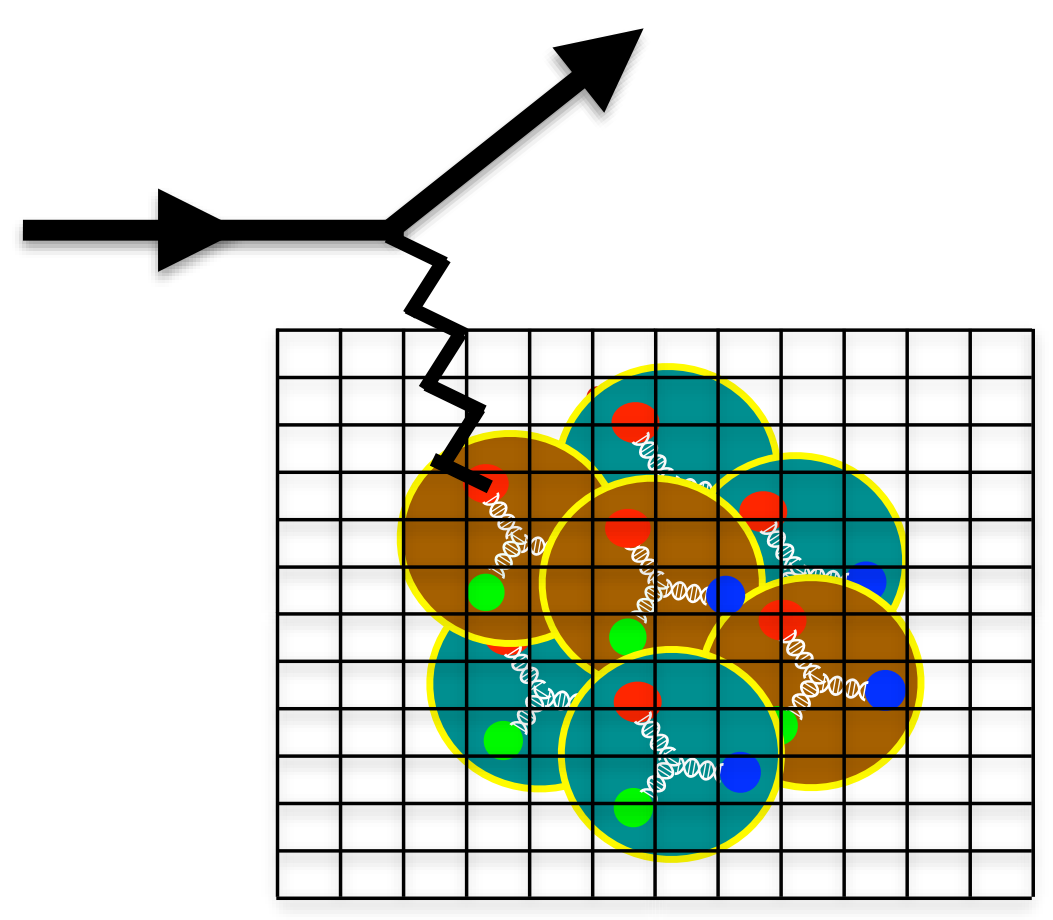

\section{Third Nuclear and Particle \\ Theory Meeting}

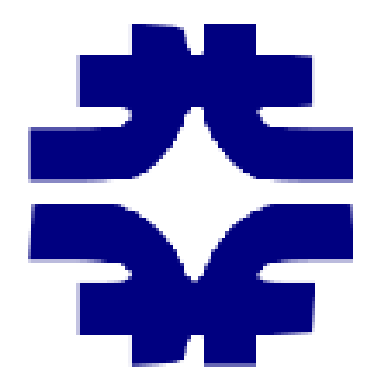

Fermilab
FERMILAB-SLIDES-21-037-T

May 11, 2020
This manuscript has been authored by

Alliance, LLC under Contract No. DE-

AC02-07CH11359 with the U.S. Department of Energy, Office of Science, Office of High Energy Physics. 


\section{Challenges of $\nu A$}

Neutrino-argon cross-sections with few percent-level accuracy required to achieve design sensitivity to $C P$ violation at DUNE

Acciarri et al (DUNE) arXiv 1512.06148
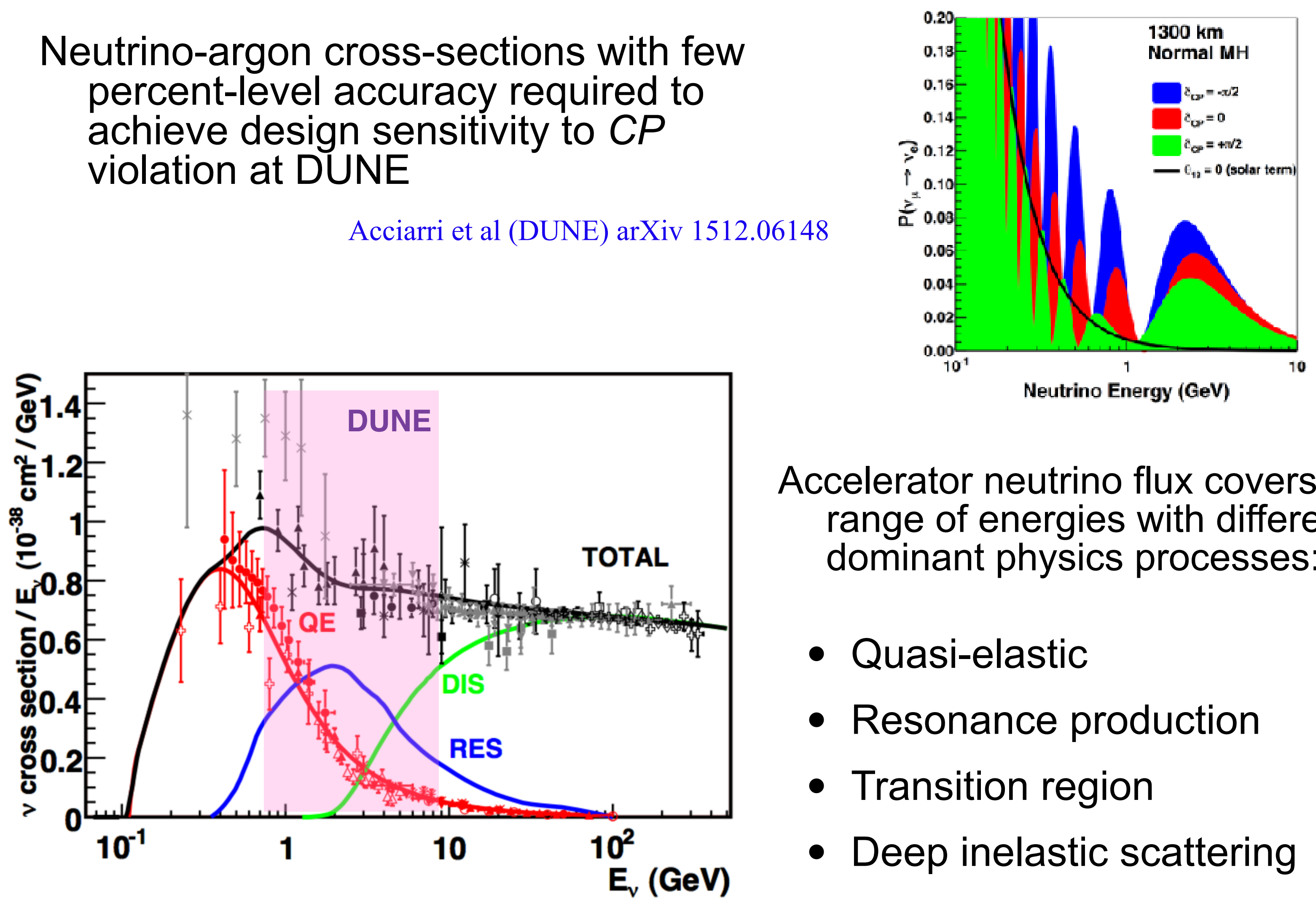

Accelerator neutrino flux covers a wide range of energies with different dominant physics processes:

- Quasi-elastic

- Resonance production

- Transition region

- Deep inelastic scattering 


\section{Lattice QCD and $\nu A$}

LQCD can provide accurate constraints on $\nu A$ scattering across energies

See USQCD $\nu A$ white paper: Kronfeld et al Eur. Phys. J. A 55 (2019)
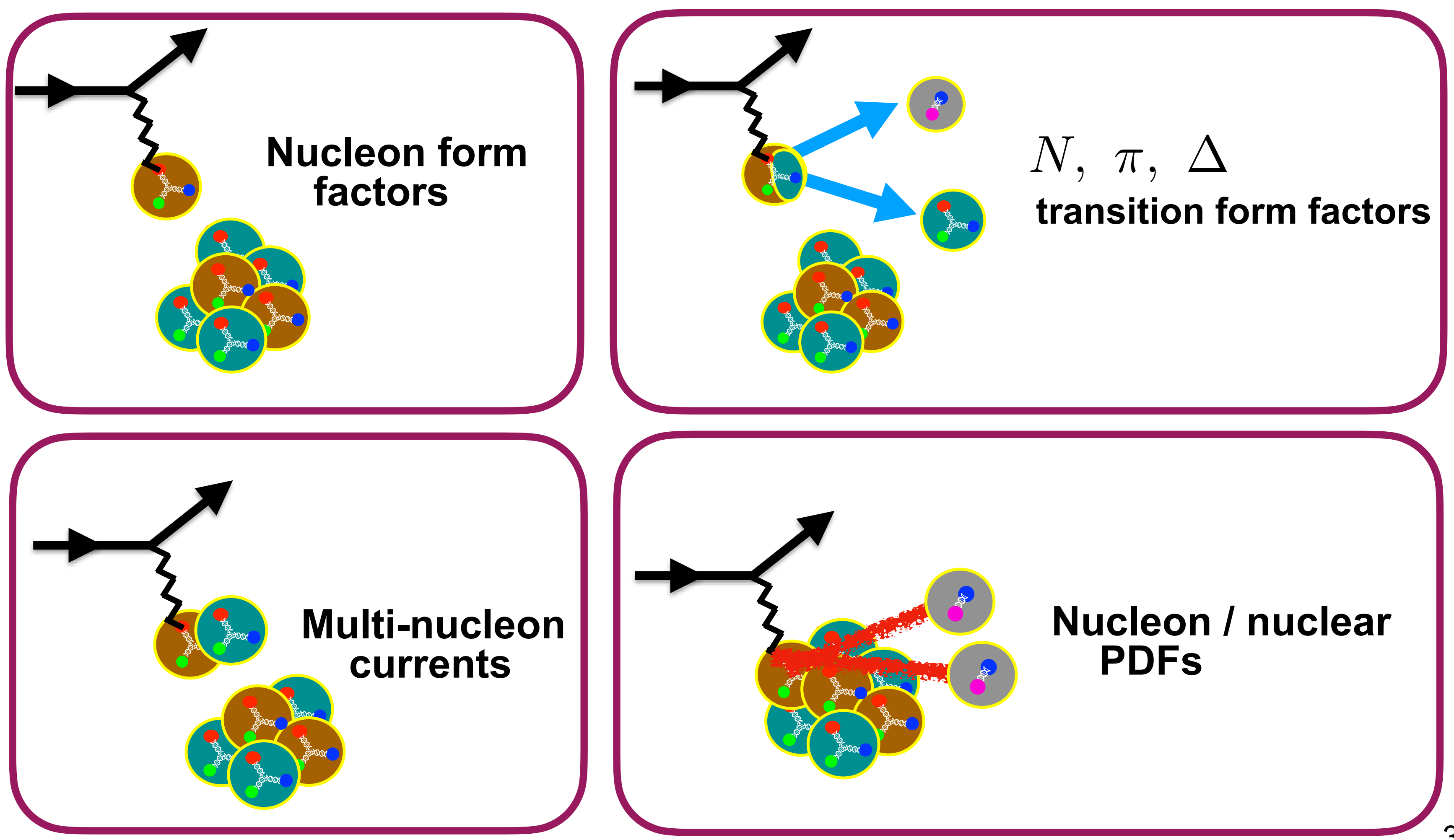

Nucleon / nuclear PDFs 


\section{Complementarity with experiment}

Easy for LQCD

Matrix elements of local operators with

spacelike momenta

Flavor dependence

(neutrons $\sim$ protons)

Spin dependence

(axial vector)

Pions
Hard for LQCD

Real-time scattering amplitudes above multi-particle production thresholds

Large baryon number

LQCD can't predict high-energy nuclear x-secs on it's own

LQCD can provide inputs to nuclear models that complement experiment and validate models of transition between EFT and PQCD 


\section{The hadron tensor}

$\nu A$ cross-section factorizes into product of lepton and hadron tensors

$$
\frac{d^{2} \sigma}{d E^{\prime} d \Omega} \propto L_{\mu \nu} W^{\mu \nu}
$$

Lepton tensor calculable with electroweak perturbation theory, hadron tensor includes nonperturbative QCD effects. For generic hadron $h$

$$
\begin{aligned}
W_{\mu \nu}^{(h)}(q, p) & =\int \frac{d^{4} x}{4 \pi} e^{i q \cdot x}\left\langle h(p)\left|J_{\mu}(x) J_{\nu}(0)\right| h(p)\right\rangle \\
& =\int \frac{d^{4} x}{4 \pi} e^{i q \cdot x} \sum_{f}\left\langle h(p)\left|J_{\mu}(x)\right| f\right\rangle\left\langle f\left|J_{\nu}(0)\right| h(p)\right\rangle
\end{aligned}
$$

Nucleon hadron tensor includes sum over all intermediate states, could inform nuclear models of resonance, transition, and DIS regions

LQCD calculations of nucleon hadron tensor under study, challenging due to inverse Laplace transform needed to relate Euclidean and Minkowski 


\section{Nuclei and nucleons}

Hadron tensor can also be decomposed into sum over exclusive channels

$$
\left\langle f(p+q)\left|J_{\nu}(q)\right| h(p)\right\rangle
$$

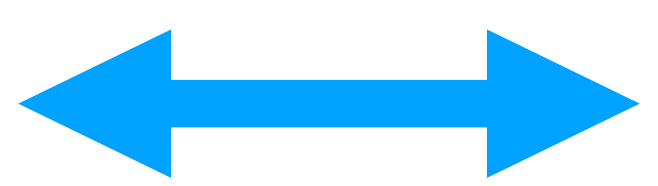

$$
W_{\mu \nu}^{(h)}(q, p)
$$

At low energies where nucleons are effective degrees of freedom, nuclei look like sums of nucleons plus corrections from multi-nucleon currents
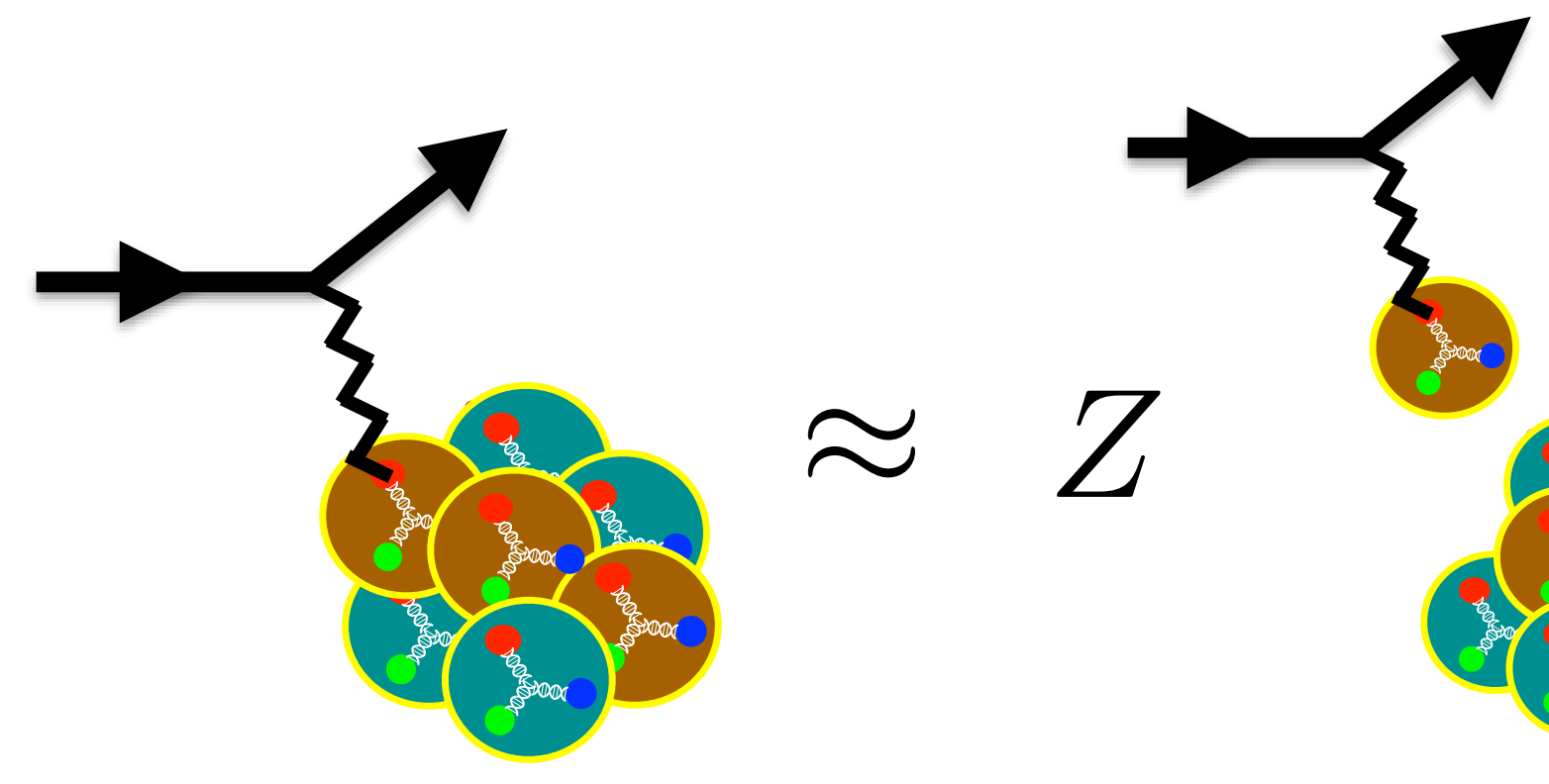

$$
+(A-Z)
$$

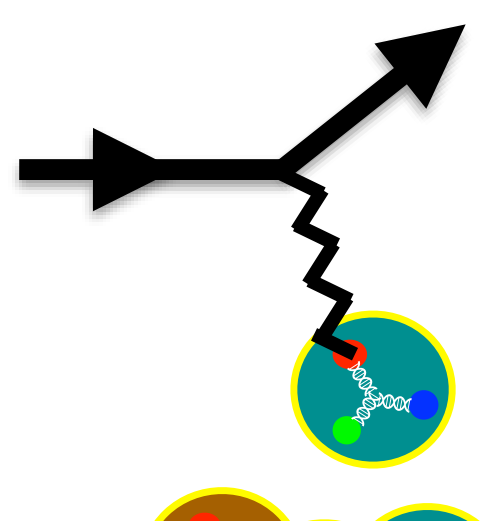

Nucleon elastic form factors are key inputs to nuclear EFTs / models

Vector form factors known from electron scattering, axial are less constrained

$$
\left\langle N(p+q)\left|A_{\mu}(q)\right| N(p)\right\rangle=\bar{u}(p+q)\left[G_{A}\left(Q^{2}\right) \gamma_{\mu} \gamma_{5}+\frac{\widetilde{G}_{P}\left(Q^{2}\right)}{2 M_{N}} q_{\mu} \gamma_{5}\right] u(p)
$$




\section{Nucleon form factors}

Vector and axial form factors recently calculated using nearly physical quark masses:
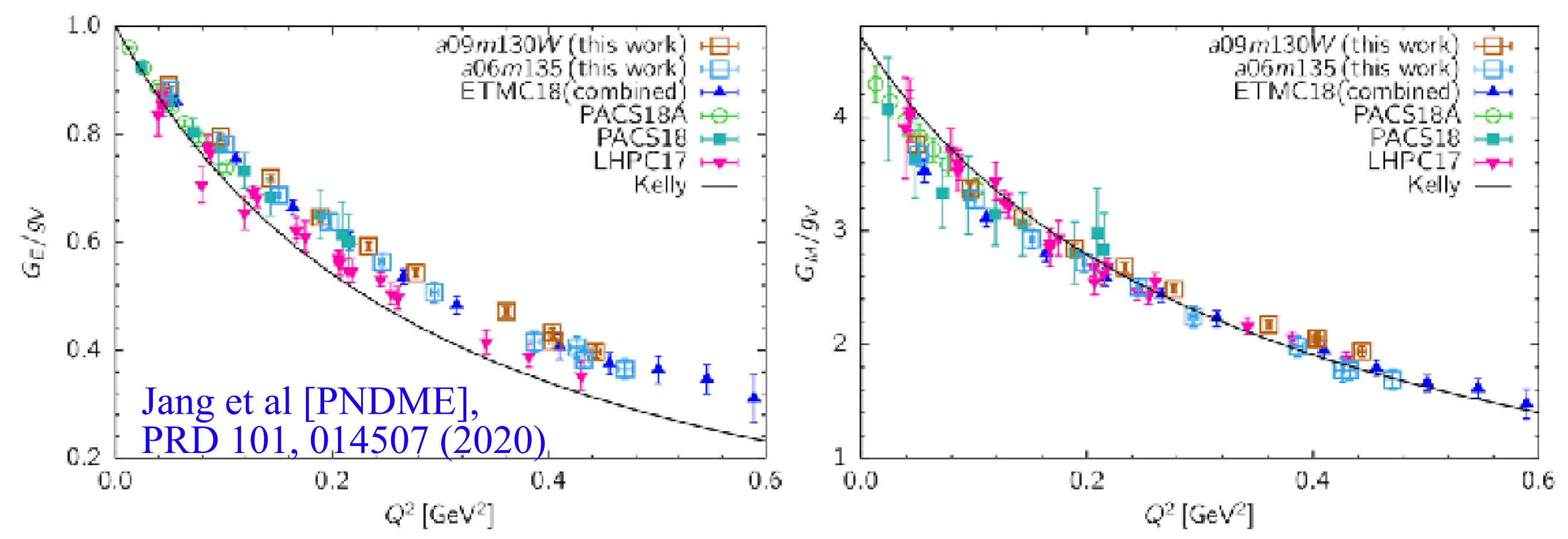

Remaining LQCD systematic uncertainties arise from lattice spacing effects, finite-volume effects, excited-state effects

Recent vector / axial form factor studies show the importance of $N \pi$ excited-state effects in nucleon form factor calculations with light quark masses
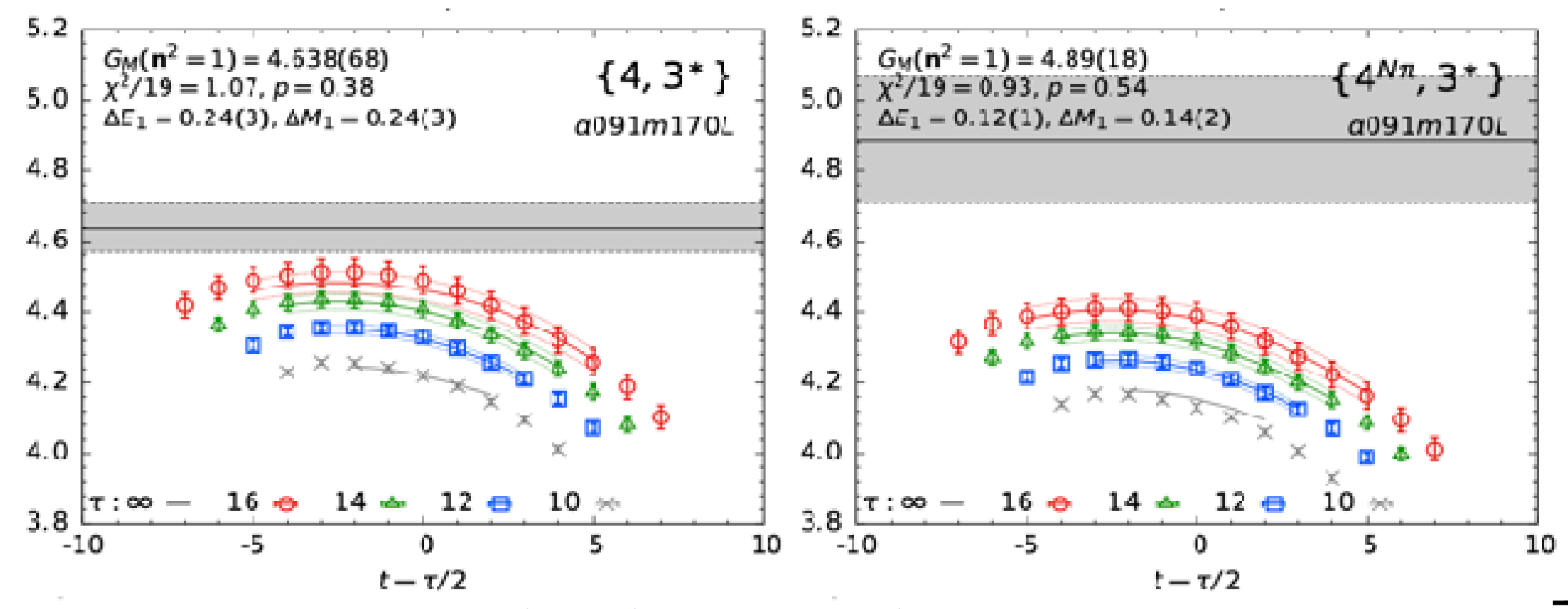

Park et al [NME], arXiv:2103.05599 


\section{Resonance production}

$\pi$ production essential for reproducing cross-section at energies above quasi-elastic region

$E_{c}=961 \mathrm{MeV}, \theta_{\mathrm{e}}=37.5^{\circ}$

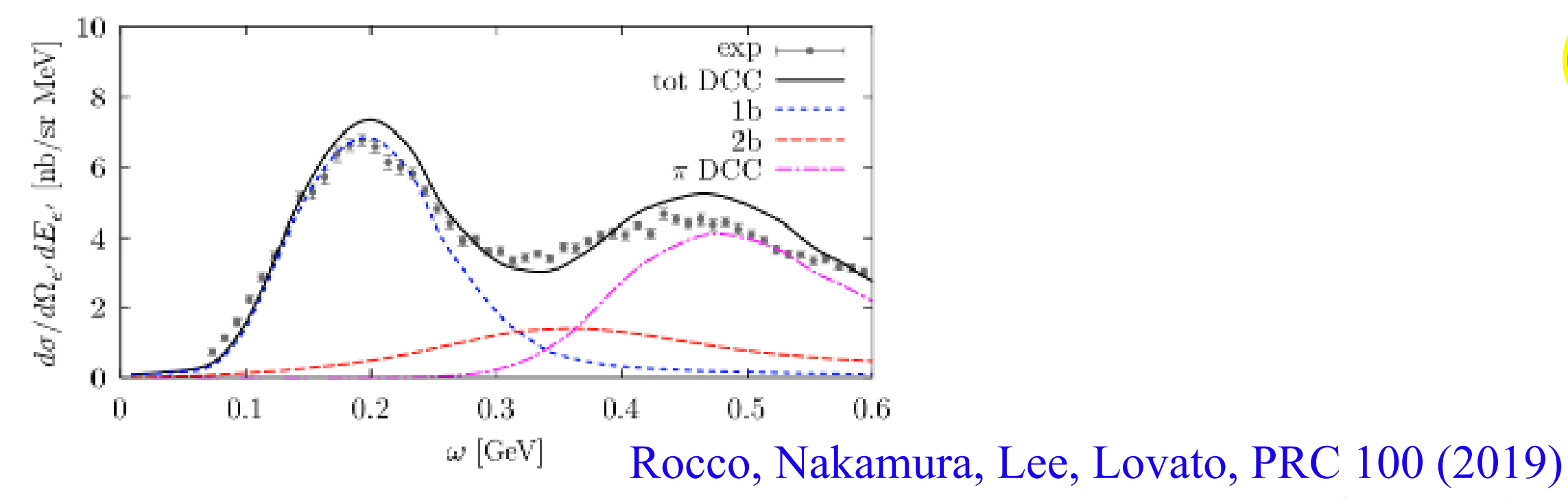

$\Delta$ resonance contributions important and poorly known ingredient to nuclear models of resonance region

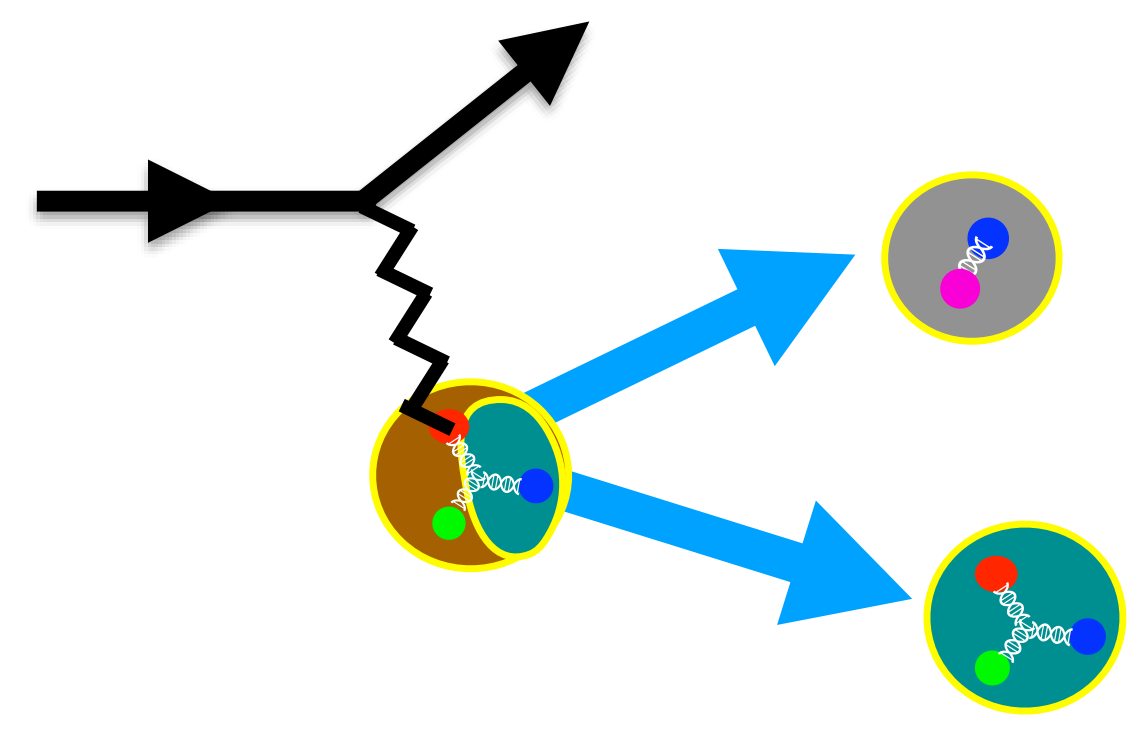

Exploratory LQCD calculations of $N, \pi, \Delta$ performed at heavy quark masses where $\Delta$ stable

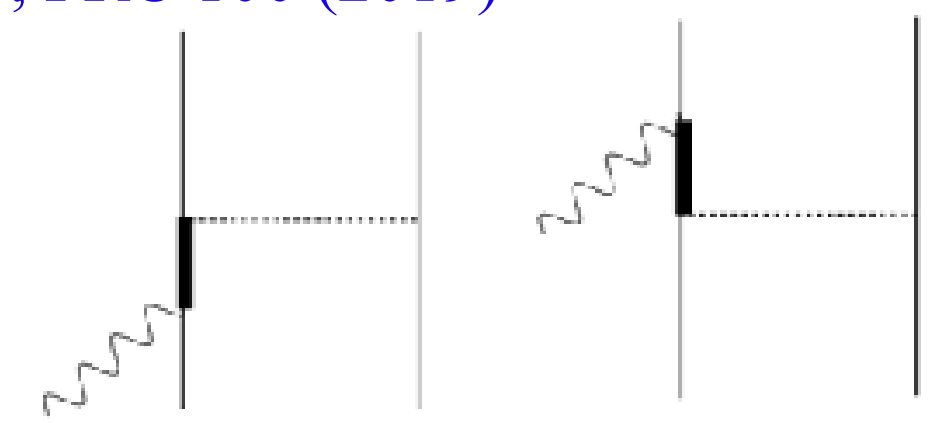

Leinweber, Draper, Woloshyn PRD 48 (1993)

$\cdots$

Alexandrou et al, PRD 77 (2008)

Fully controlled LQCD calculations at physical point require sophisticated finite-volume formalism and numerical techniques for $N \pi$ scattering states 


\section{$N \pi$ scattering}

Recent calculations in $I=3 / 2$ channel including $\Delta$ and $N \pi$ interpolating operators use variational methods to extract ground- and excited-states in many angular momentum channels (cubic irreps)

Silvi, Paul, Alexandrou, Krieg, Leskovec, Meinel, Negele, Petschlies, Pochinsky, Rendon, Syritsyn, Todaro, arXiv:2101.00689

Finite-volume quantization conditions relate energy levels to $\mathrm{p}$-wave $N \pi$ scattering phase shifts
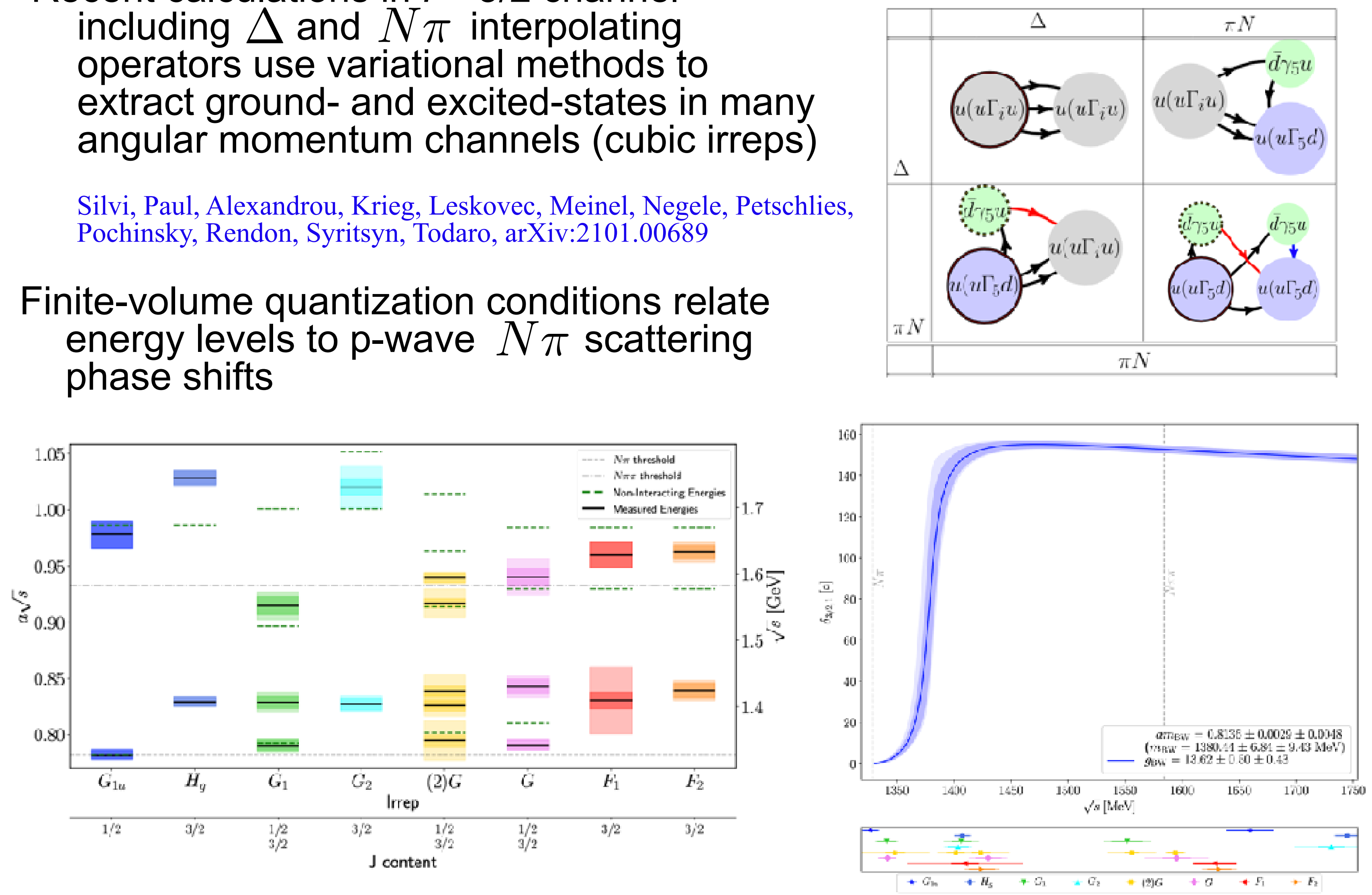


\section{LQCD and PDFs}

At very high energies, cross-sections factorize into convolutions of perturbative quark-and-gluon-level amplitudes and nonperturbative PDFs

Euclidean matrix elements containing hard scales can be factorized into perturbative coefficients and structure functions

Large momentum effective theory review: Ji et al, arXiv:2004.03543

For colinear PDFs and some aspects of TMDPDFs, Euclidean and lightcone structure functions are related by Lorentz invariance
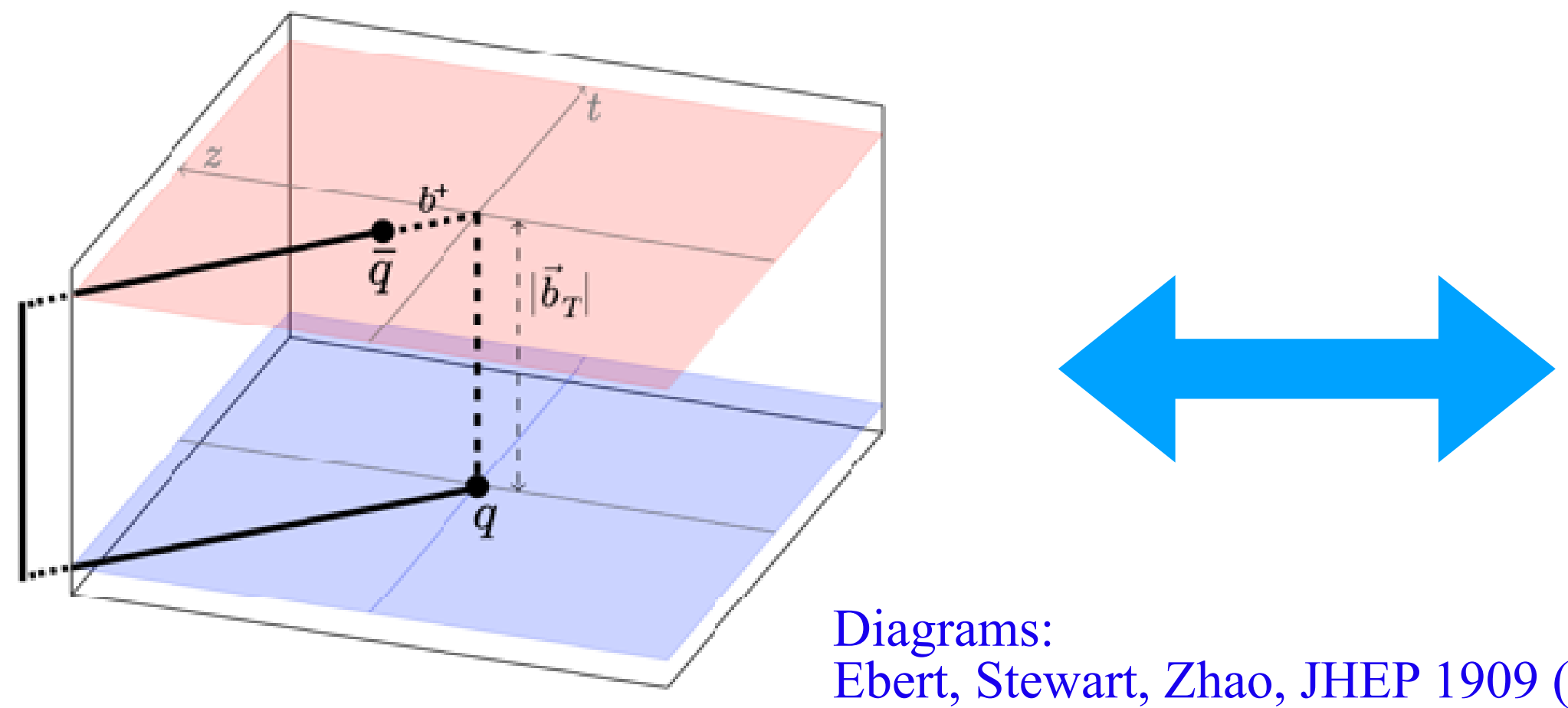

Diagrams:

Ebert, Stewart, Zhao, JHEP 1909 (2019)

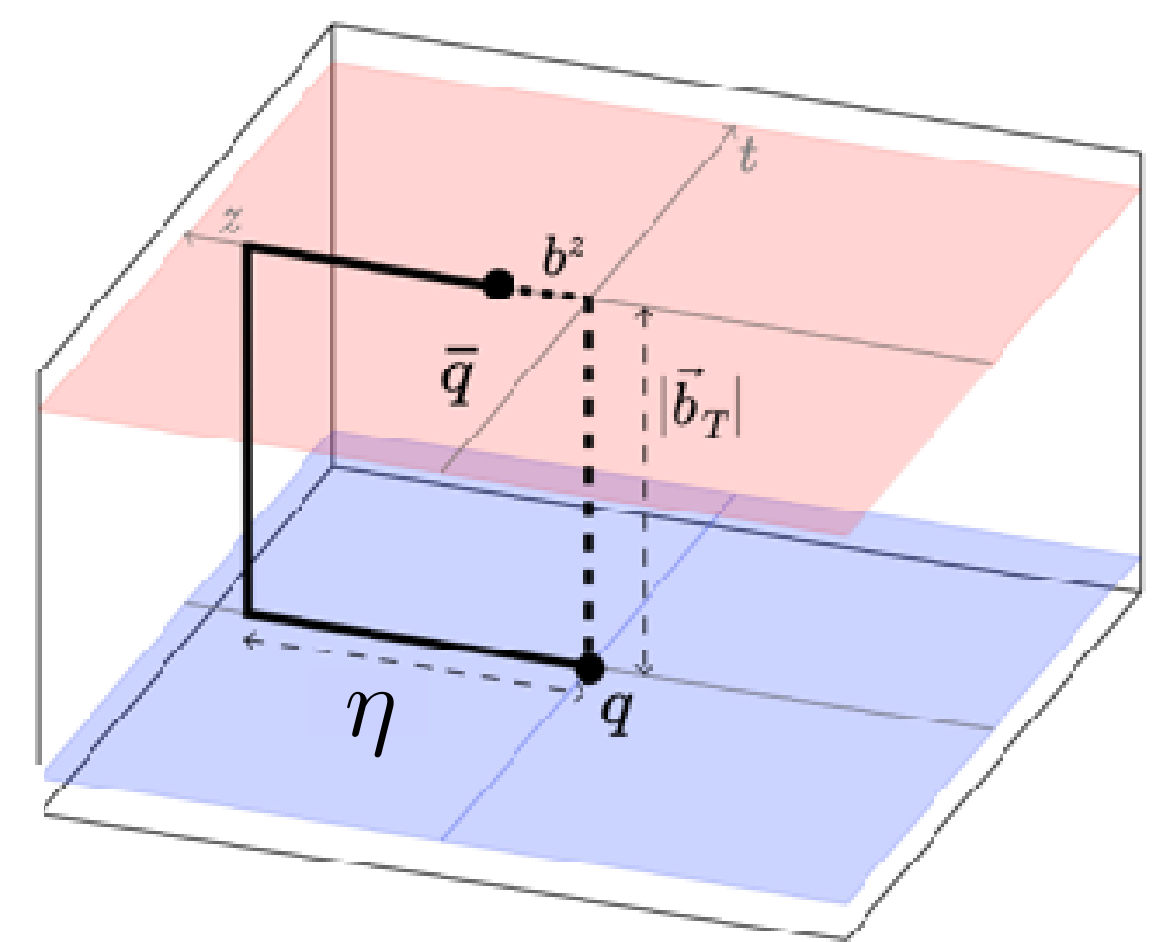

High-energy axial current interactions depend on polarized PDFs, SIDIS process like high-energy $\mathrm{CC} 1 \pi$ depend in principle on TMDPDFs, ... 


\section{Nucleon PDFs}

LQCD calculations of unpolarized nucleon PDFs are maturing but still face challenges (e.g. non-local operator renormalization)

CITEME

LQCD is starting to constrain on less well-known aspects of PDFs such as flavor dependence, spin-dependence, transversity, and nuclear effects

Review: CITEME

Current LQCD results can improve global analyses of isovector polarized PDFs
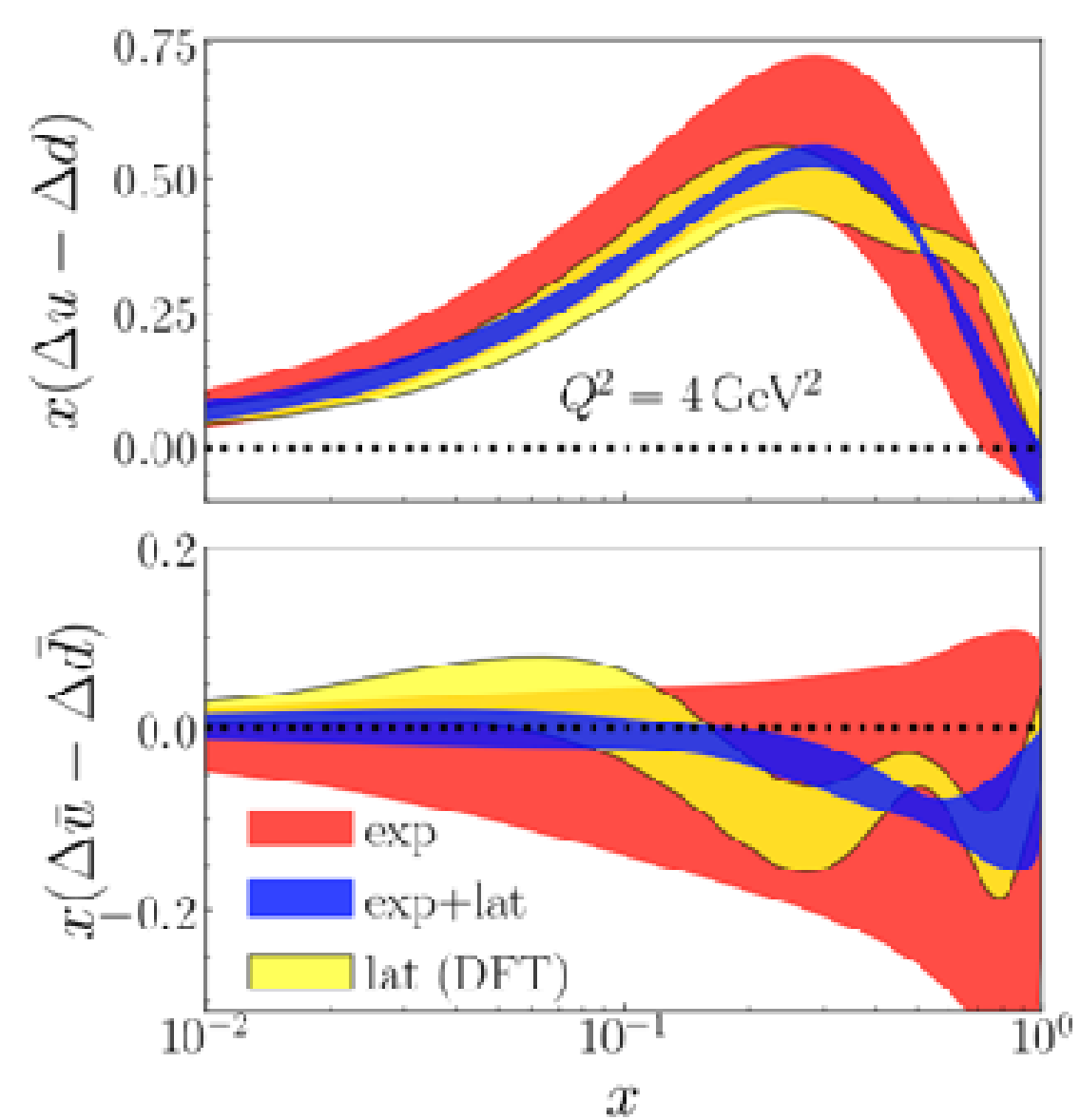

Bringewatt et al [JAM], arXiv:2010.00548
LQCD will constrain TMDPDF evolution by determining Collins-Soper kernel

Shanahan, MW, Zhao, PRD 102 (2020)

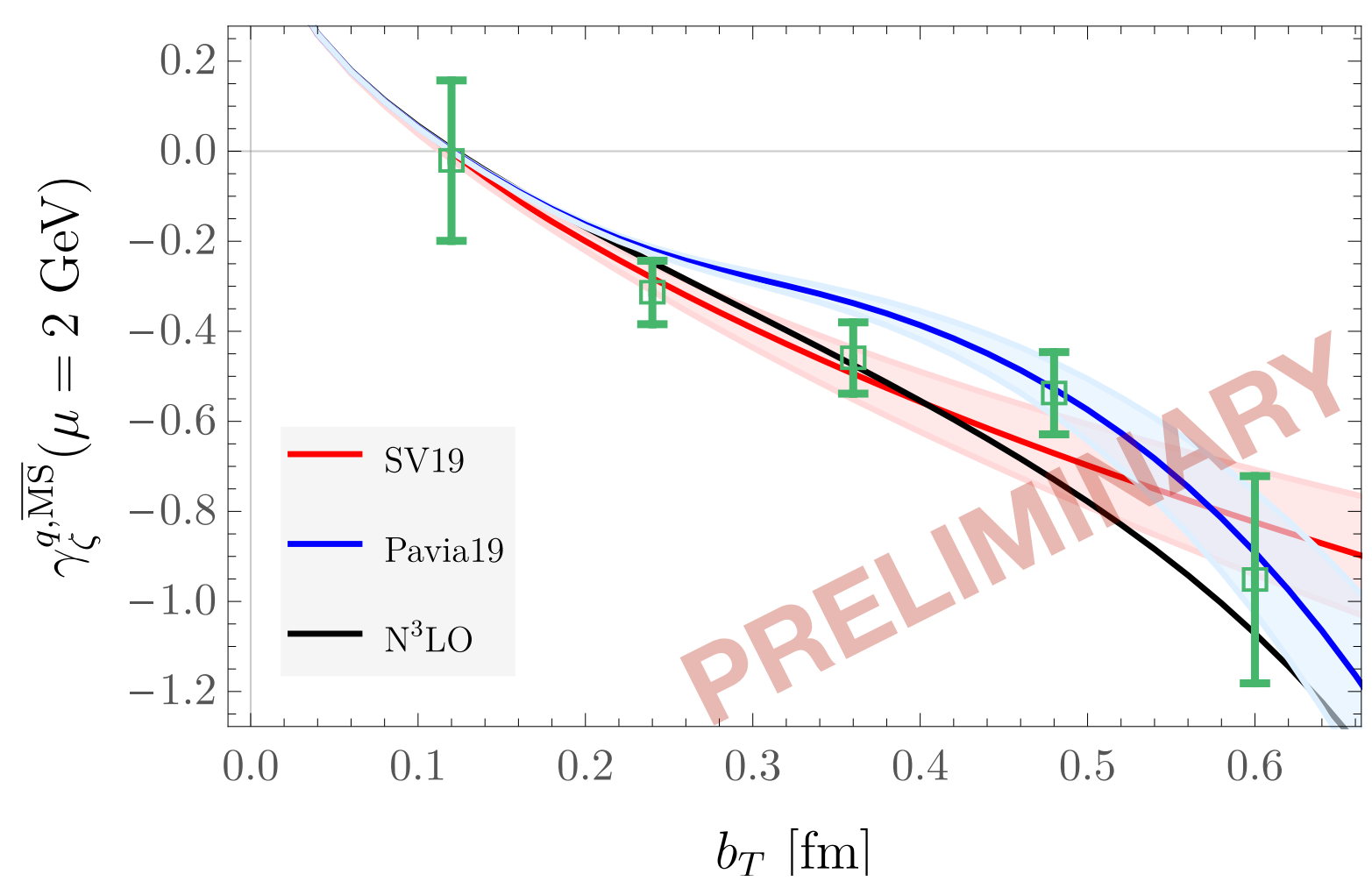

Shanahan, MW, Zhao, in preparation 


\section{Many-quark bound states}

The partonic structure of nuclei is noticeably different from the nucleon

Aubert et al (EMC), Phys. Lett. 123B (1983)

The EMC effect and it's analogs can be described by two-body currents in EFT

CITEME

Emergence from QCD is not yet understood
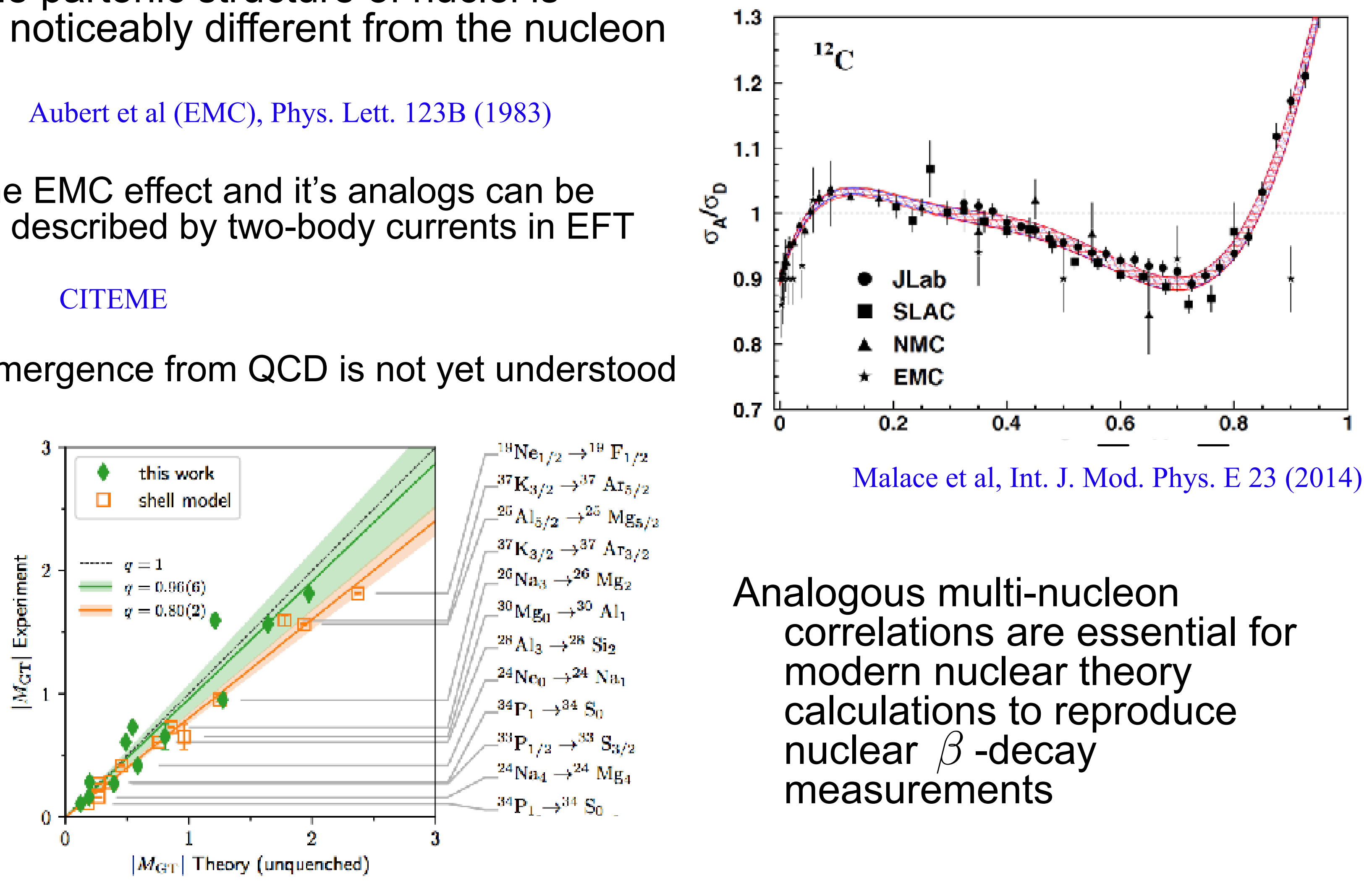

Analogous multi-nucleon correlations are essential for modern nuclear theory calculations to reproduce nuclear $\beta$-decay measurements

Gysbers et al, Nature Phys. 15 (2019) 


\section{Quarks in ${ }^{3} \mathrm{He}$}

Momentum fraction carried by any quark flavor (or gluons) calculable from local matrix elements in generic hadrons

$$
\langle x\rangle_{h}^{i}=\int_{-1}^{1} d x x q_{h}^{i}(x)=C\left(M_{h}, \vec{p}, J\right) \sum_{\lambda=-J}^{J}\left\langle h, \vec{p}, \lambda\left|T_{\mu \nu}^{i}\right| h, \vec{p}, \lambda\right\rangle
$$

First calculation of isovector quark momentum fractions of light nuclei

Detmold, MW et al [NPLQCD] PRL.xx

arXiv:2009.05522

Although systematic uncertainties are not fully controlled (one lattice spacing, volume, quark mass, ...) demonstrates potential for LQCD to usefully constrain nuclear PDFs
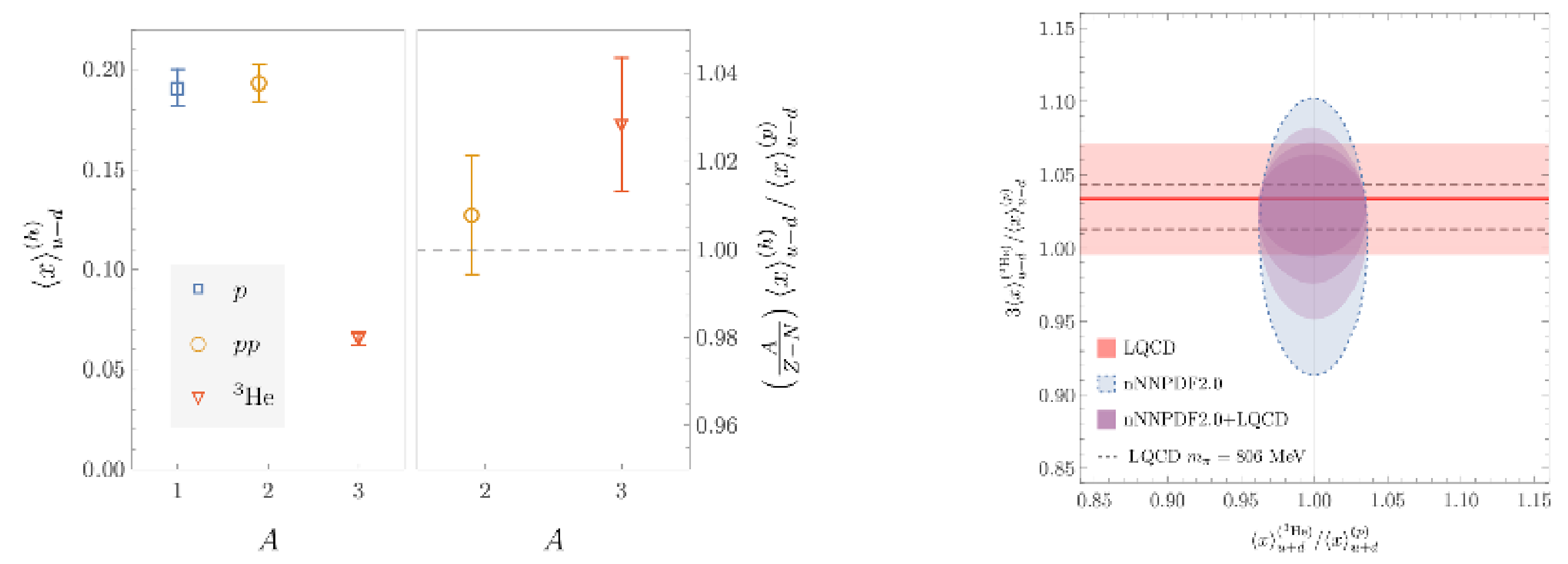


\section{Two-body currents}

Constraints on two-body axial currents obtained by matching LQCD and EFT calculations in a box with a background axial field
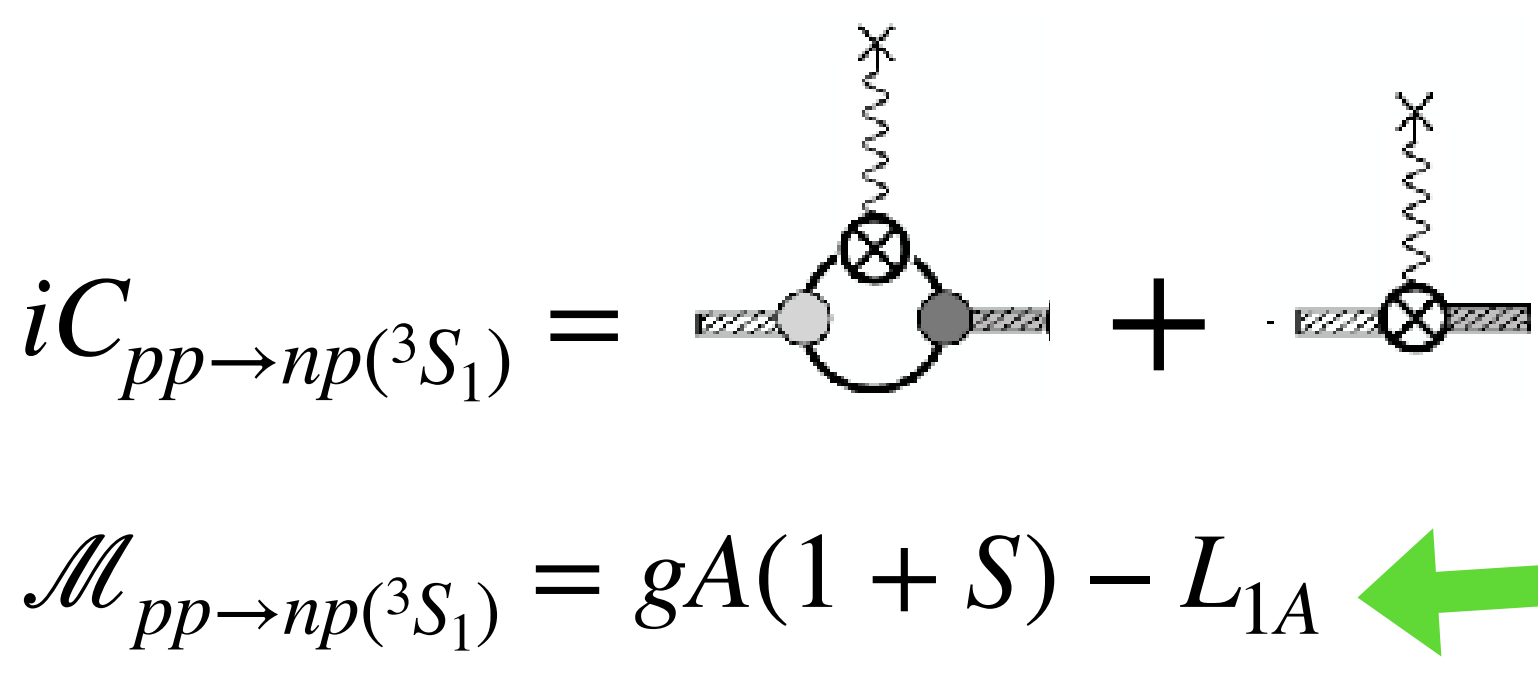

Short-distance

QCD physics

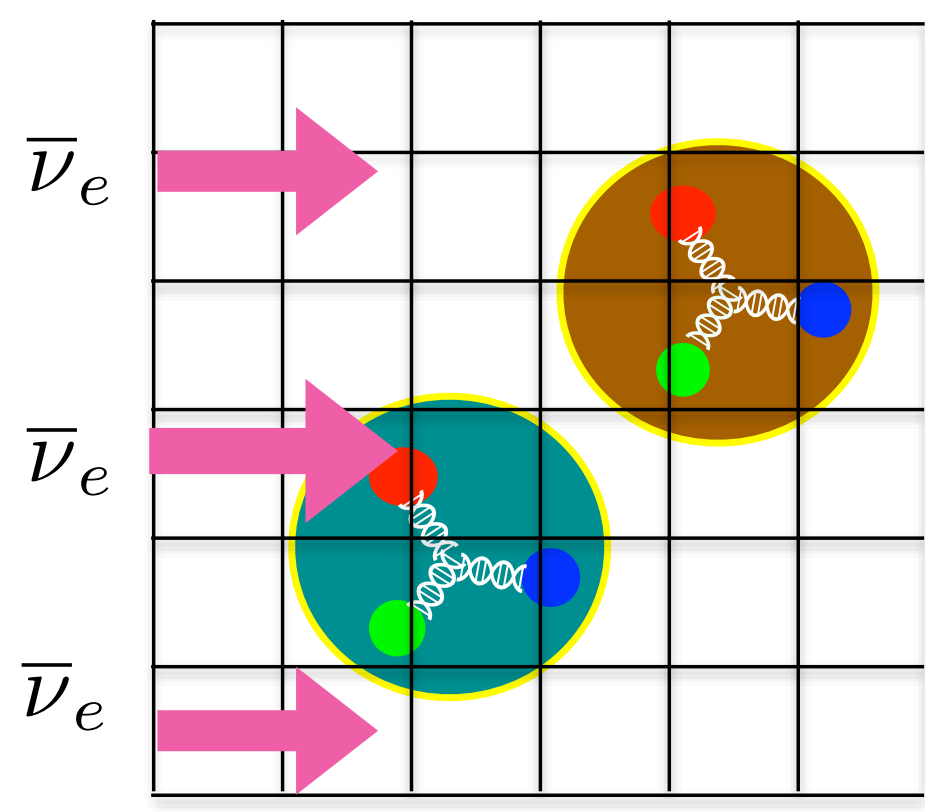

First exploratory LQCD determination of $L_{1 A} \quad$ Savage, MW et al [NPLQCD], PRL 119 (2017)

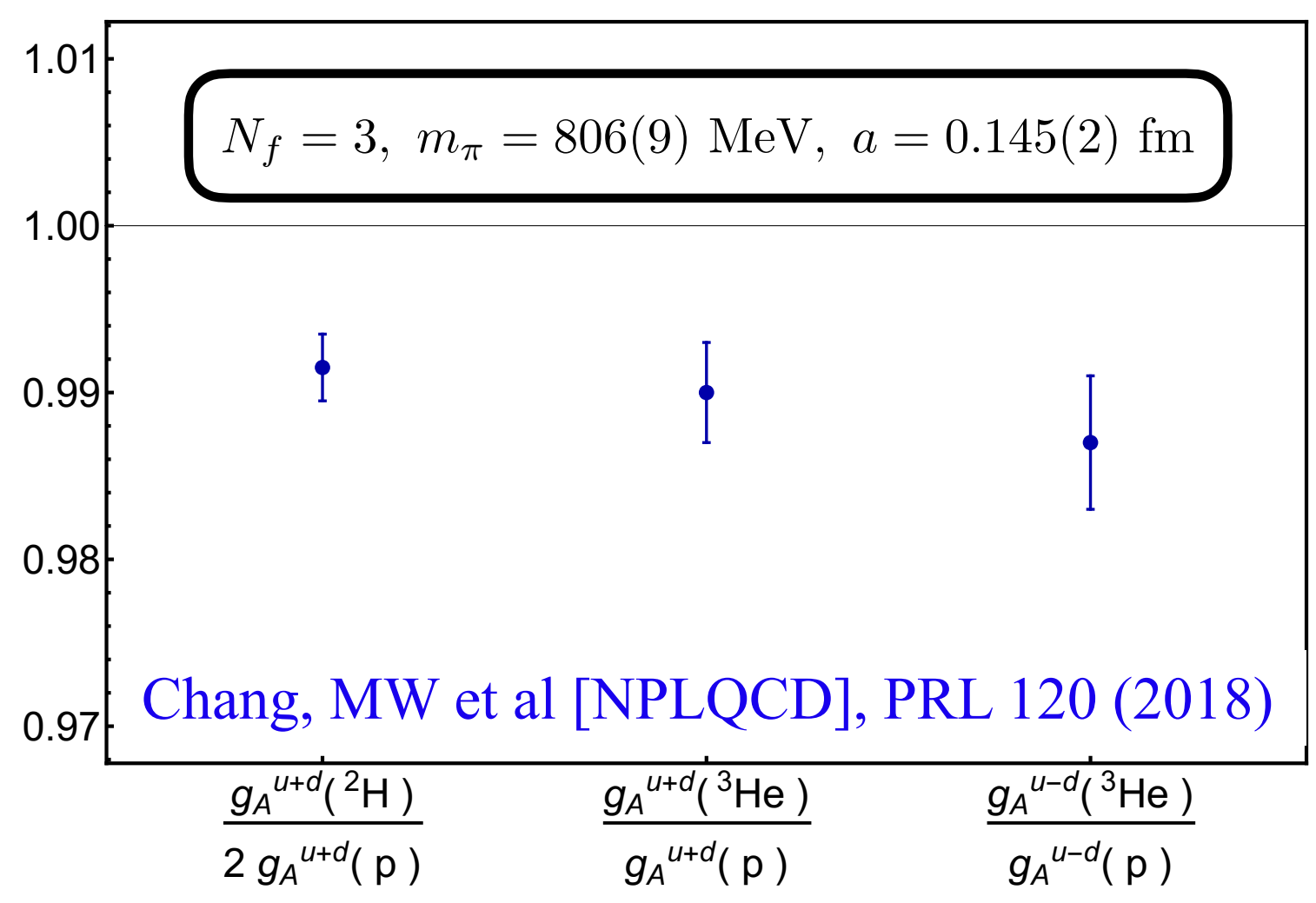

LQCD calculations of $A=2-3$ matrix elements sensitive to two-body currents performed at unphysically heavy quark masses 


\section{Triton $\beta$-decay}

Triton $\beta$ - decay Gamow-Teller matrix elements calculated with two quark mass values, permits first extrapolation to physical masses

Several systematic uncertainties remain, but encouraging agreement with experiment seen

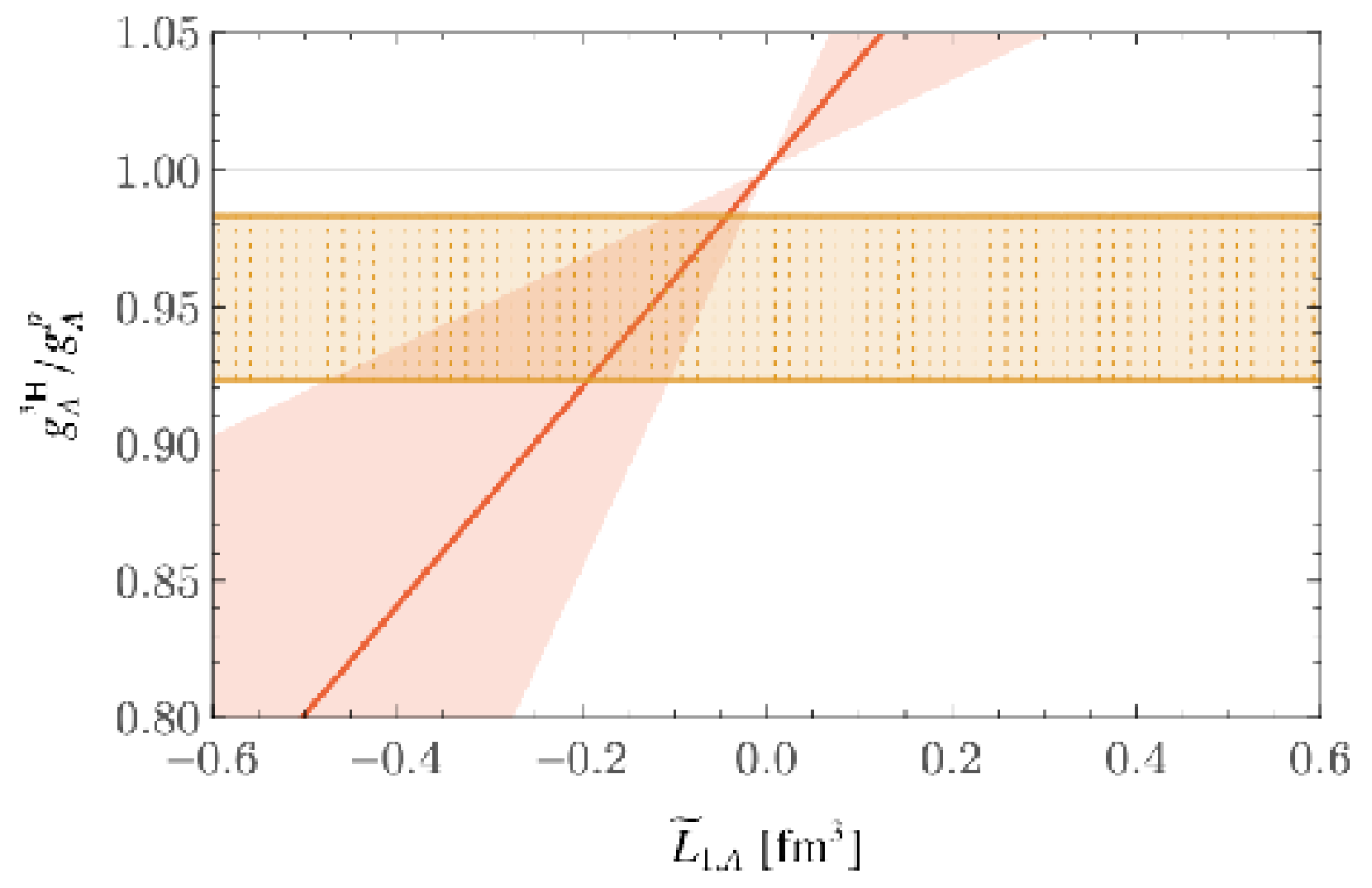

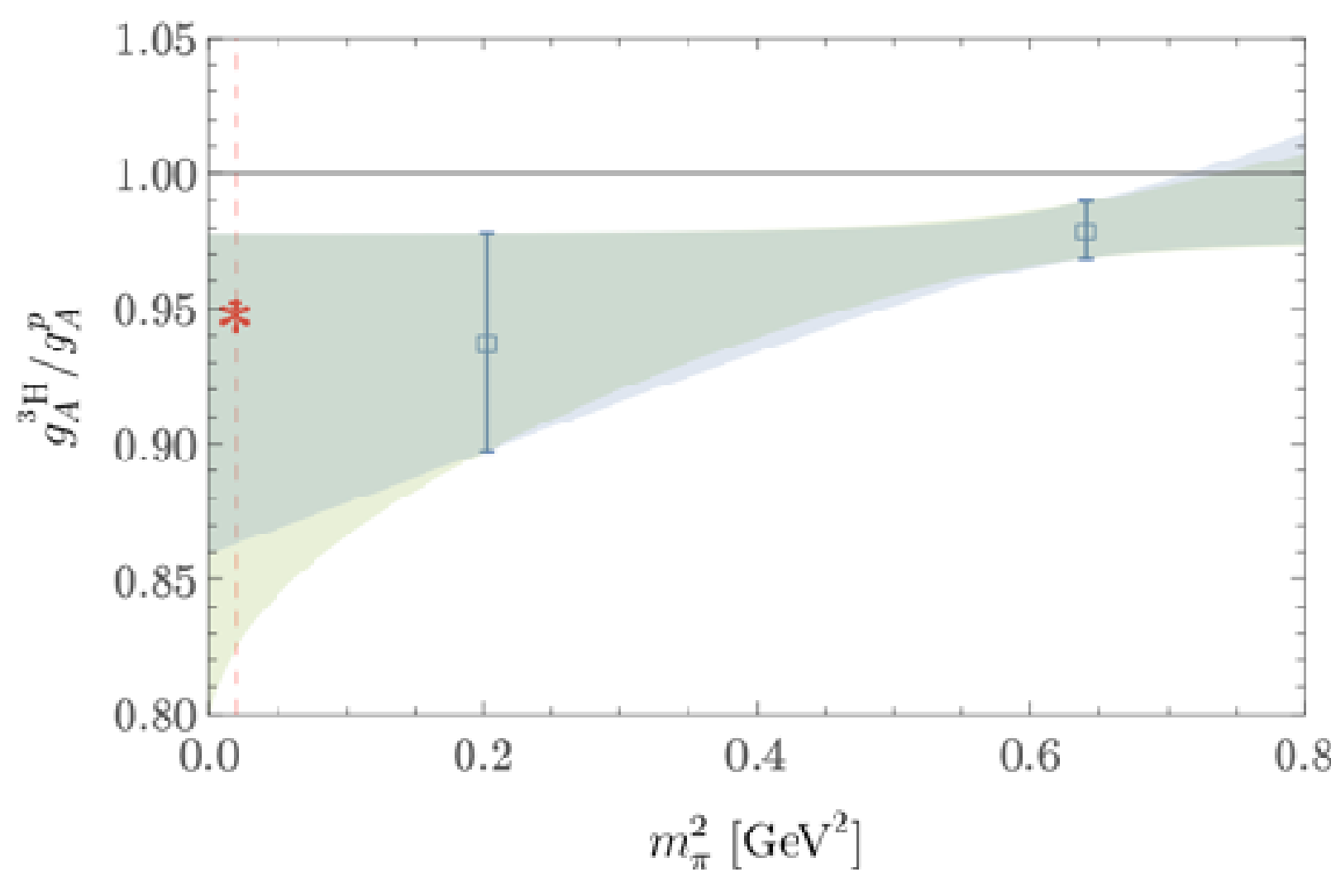

Parreño, MW et al [NPLQCD] PRD 103 (2021)

Results used to constrain two-body axial current in pionless EFT

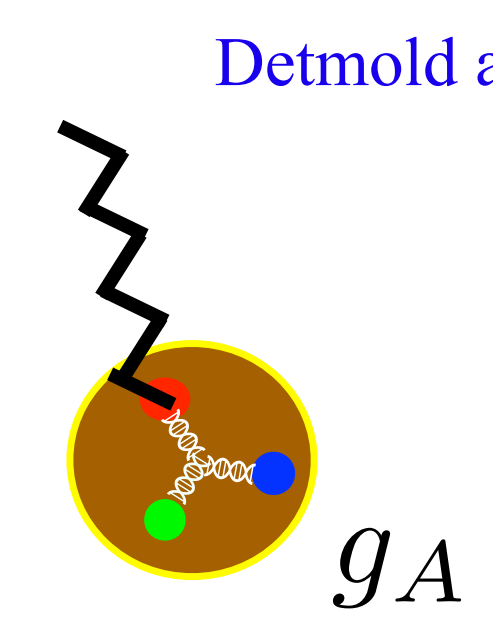




\section{Systematic uncertainties}

Several systematic uncertainties of nuclear matrix element calculations still need to be quantified in detail

- Heavier than physical quark masses only

- One lattice spacing

- Excited-state effects

Gap between ground and finite-volume "scattering" states becomes small for large volumes, ground-state dominance relies on overlap factors

$$
Z_{0} e^{-E_{0} t}\left(1+\frac{Z_{1}}{Z_{0}} e^{-\delta t}+\ldots\right) \quad \delta \sim \frac{4 \pi^{2}}{M L^{2}}
$$

Opposite-sign cancellations between the ground and excited-state could in principle conspire to form a "false plateau"

See e.g. Iritani et al, JHEP 10 (2016)

First two-nucleon LQCD calculations using positive-definite correlation functions (enabled by distillation / stochastic LapH) show significant interpolating operator and lattice spacing dependence 


\section{Interpolating operators}

Composite quark \& gluon operators produce all QCD energy eigenstates with appropriate quantum numbers

$$
C_{\chi \chi^{\prime}}^{\left(B, I, \Gamma_{J}\right)}(t)=\left\langle\chi^{\left(B, I, \Gamma_{J}\right)}(x) \bar{\chi}^{\left(B, I, \Gamma_{J}\right)}(0)\right\rangle=\sum_{\mathbf{n}} Z_{\mathbf{n} \chi}^{\left(B, I, \Gamma_{J}\right)}\left(Z_{\mathbf{n} \chi^{\prime}}^{\left(B, I, \Gamma_{J}\right)}\right) e^{-t E_{\mathrm{n} \chi}^{\left(B, I, \Gamma_{J}\right)}}
$$

Full spectrum in principle encoded in $t$ dependence of any correlation function

With finite numerical data, inverting to obtain energies is ill-posed in general

To isolate ground state, would ideally use

$$
t \gg \frac{1}{E_{1}^{\left(B, I, \Gamma_{J}\right)}-E_{0}^{\left(B, I, \Gamma_{J}\right)}}
$$

- Signal-to-noise problem prevents this from being achievable

Alternative - construct operators with

$$
Z_{0 \chi}^{\left(B, I, \Gamma_{J}\right)} \gg Z_{\mathrm{n} \chi}^{\left(B, I, \Gamma_{J}\right)}
$$

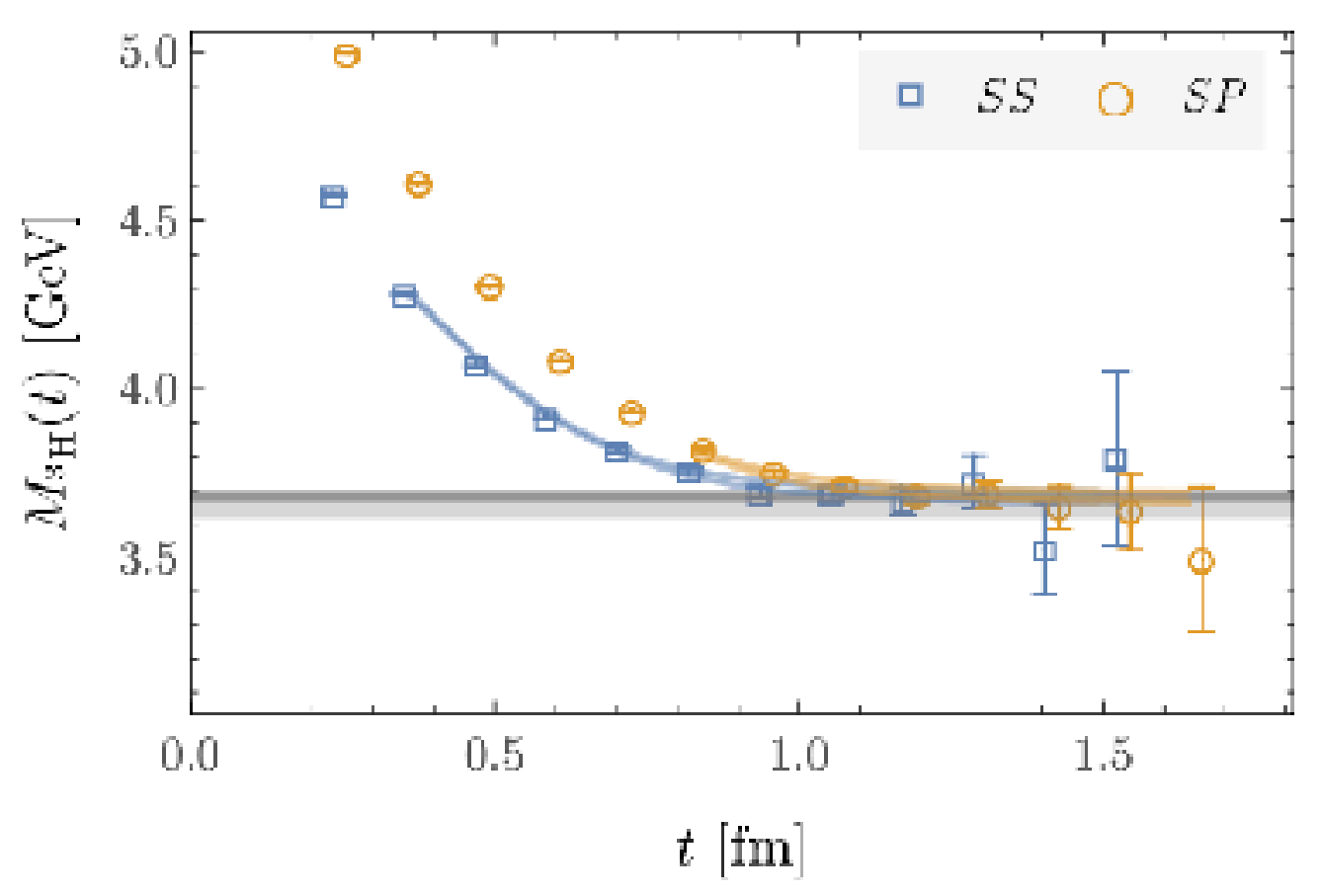




\section{Variational LQCD methods}

Interpolating operator sets including both local "hexaquark" and plane-wave "dibaryon" operators generalize the sets used in previous calculations
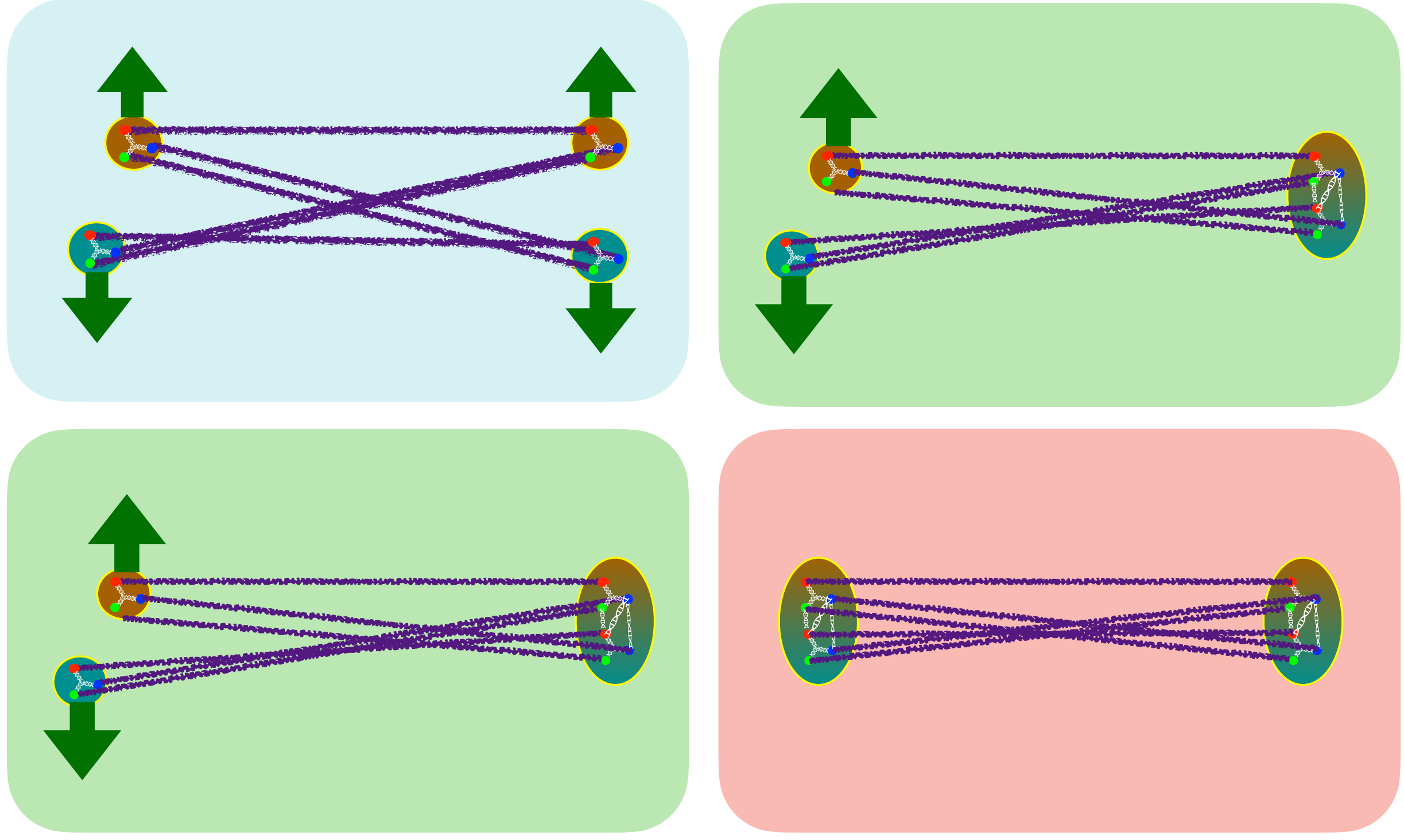

Analogous variational methods have been successfully applied in meson channel, technically challenging for two-nucleon systems

New variational calculations enabled by algorithmic and computational advances are being explored by multiple groups 


\section{$N N$ excited states}

Large interpolating operator sets, e.g. $16 \times 16$ for $\mathrm{I}=1$ spin-singlet channel

- local "hexaquark",

- two-nucleon plane-wave "dibaryon"

- two-nucleon "quasi-local" resembling EFT bound state wavefunction diagonalized to map out ground- and excited-state energy levels
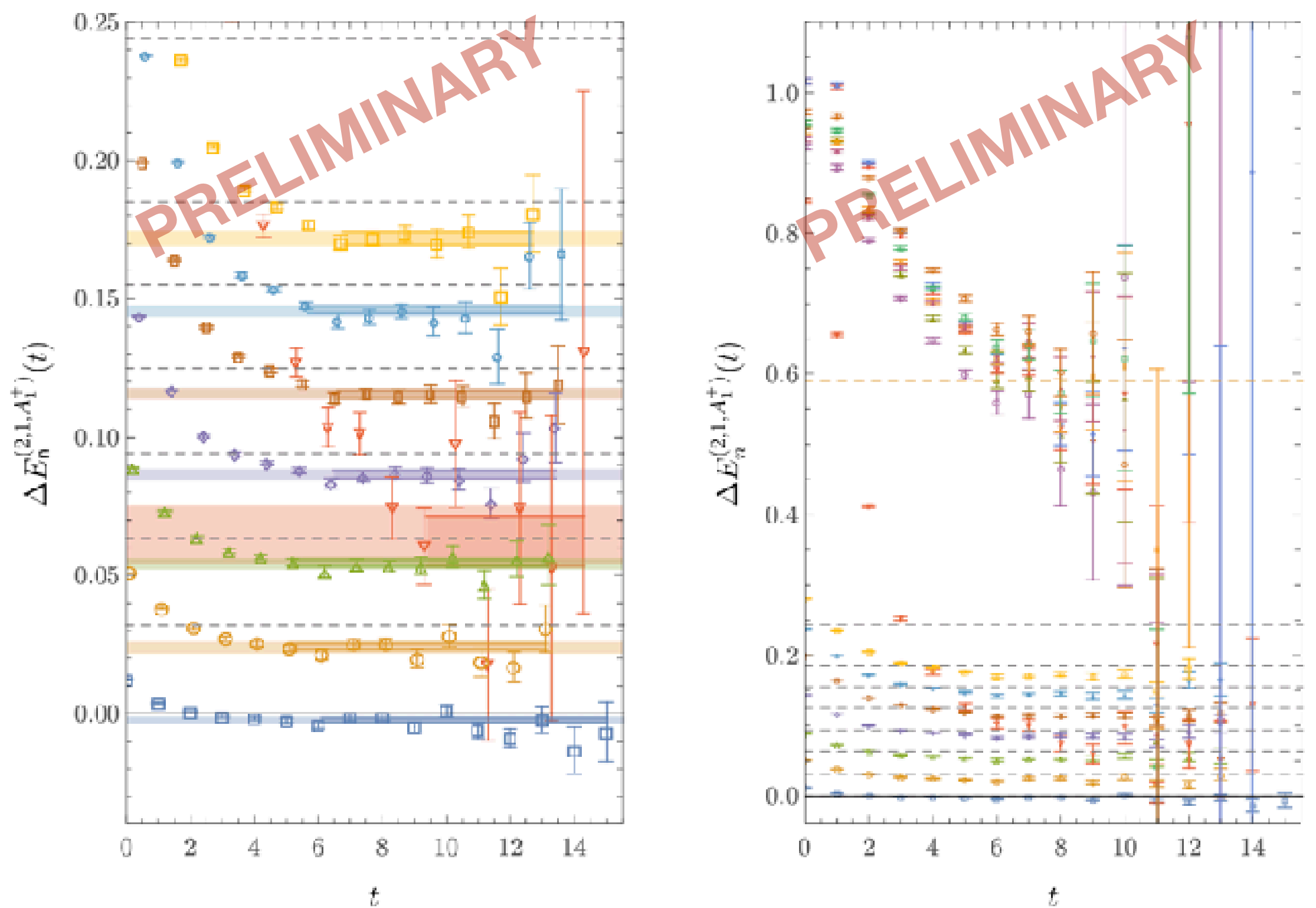


\section{Towards $N \pi$ excited states}

First calculations applying variational methods to $l=3 / 2 N \pi$ scattering have been performed

Silvi et al, arXiv:2101.00689

Significant recent progress in relating calculable finite-volume matrix elements to physical amplitudes for resonance production

See e.g. Baroni, Briceño, Hansen, Ortega-Gama, PRD 100 (2018)

Direct matching to nuclear EFT / models also possible

Variational calculations of $I=1 / 2$ nucleon

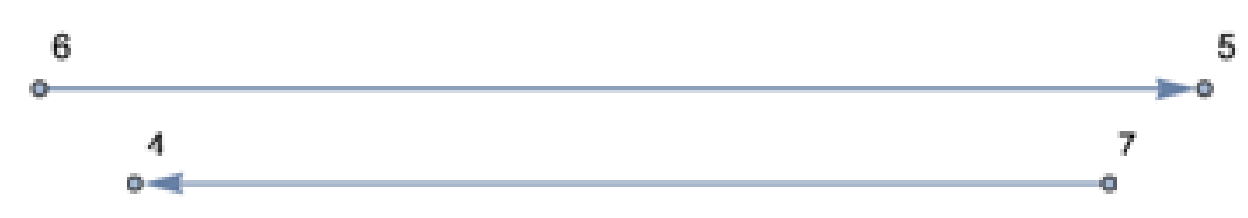
channel including explicit $N \pi$ interpolating operators and multiple lattice spacings / volumes will allow robust QCD predictions of nucleon form factors and resonance production amplitudes

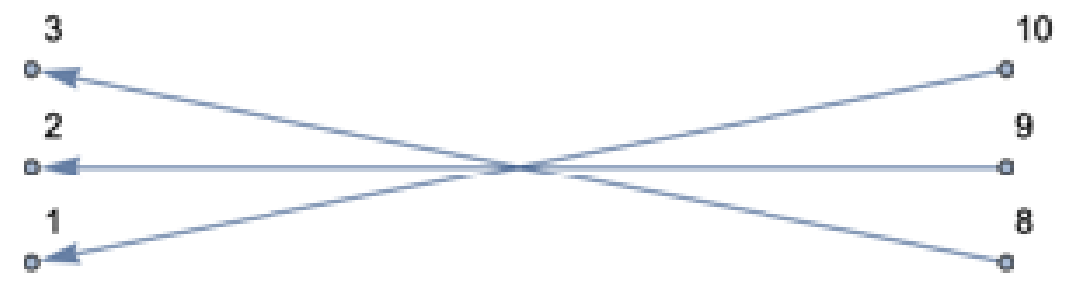




\section{Conclusions}

Describing $\nu A$ scattering from the Standard Model requires control of QCD over a wide range of scales and physics processes

Challenging but possible through lattice QCD + nuclear many body methods

Nucleon form factors
Hadron tensor
Resonance production
Two-body currents
PDF constraints

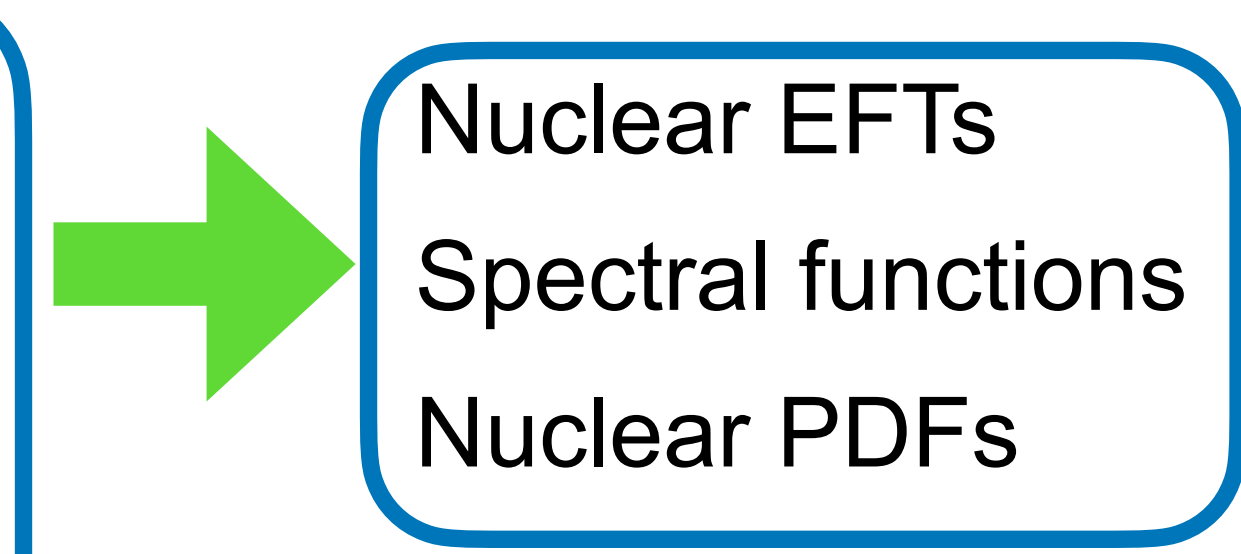

\section{Santord}

Underground

Research

Facility
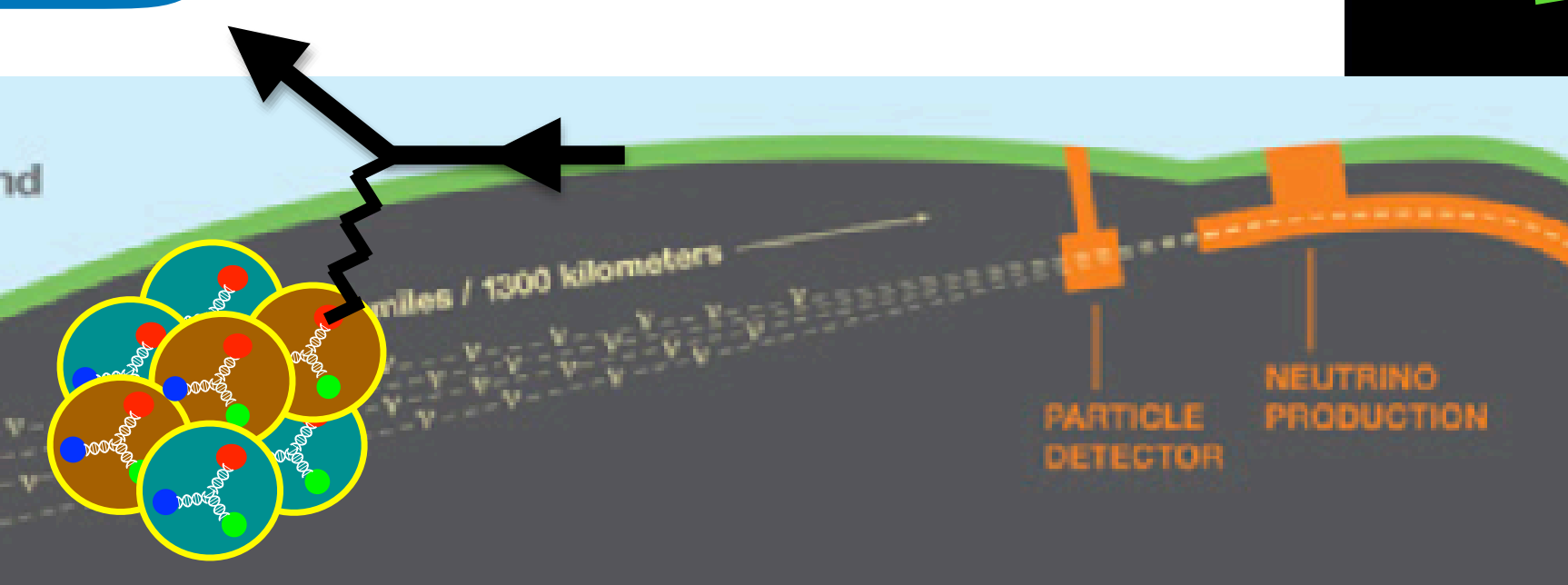

Fermilab

PARTICLE
DETECTOR 